\title{
Comparison of physical activity energy expenditure in Japanese adolescents assessed by EW4800P triaxial accelerometry and the doubly labelled water method
}

\author{
Kazuko Ishikawa-Takata ${ }^{1 *}$, Kayoko Kaneko ${ }^{2}$, Kayo Koizumi ${ }^{2}$ and Chinatsu Ito $^{3}$ \\ ${ }^{1}$ National Institute of Health and Nutrition, 1-23-1 Toyama, Shinjuku, Tokyo 1628636, Japan \\ ${ }^{2}$ Yokohama National University, 79-1 Tokiwadai, Hodogaya, Yokohama, Kanagawa 2408501, Japan \\ ${ }^{3}$ Akita Nutrition Junior College, 46-1 Sakuramorisawa Shimokitate, Akita City, Akita 0108515, Japan \\ (Submitted 16 April 2012 - Final revision received 29 January 2013 - Accepted 29 January 2013 - First published online 2 April 2013 )
}

Abstract

The present study compared the accuracy of triaxial accelerometry and the doubly labelled water (DLW) method for measuring physical activity (PA) in Japanese adolescents. A total of sixty adolescents aged 12-15 years were analysed. The total energy expenditure (TEE) was measured over $7 \mathrm{~d}$ by the DLW method and with an EW4800P triaxial accelerometer (Panasonic Corporation). The measured (RMR $\mathrm{R}_{\mathrm{m}}$ ) and predicted RMR $\left(\mathrm{RMR}_{\mathrm{p}}\right)$ were 5.7 (SD 0.9$)$ and $6.0(\mathrm{SD} 1.0) \mathrm{MJ} / \mathrm{d}$, respectively. TEE measured by the DLW method and accelerometry using $\mathrm{RMR}_{\mathrm{m}}$ or $\mathrm{RMR}_{\mathrm{p}}$ were 11.0 (SD 2.6), $10 \cdot 3$ (SD 1.9), and $10 \cdot 7$ (SD 2.1) MJ/d, respectively. The PA levels (PAL) measured by the DLW method using $\mathrm{RMR}_{\mathrm{m}}$ or $\mathrm{RMR}_{\mathrm{p}}$ were $1.97(\mathrm{SD} 0.31$ ) and 1.94 (SD 0.31) in subjects who exercised, and 1.85 (SD 0.27) and 1.74 (SD 0.29) in subjects who did not exercise. The percentage of body fat correlated significantly with the percentage difference between $\mathrm{RMR}_{\mathrm{m}} v$. RMR $\mathrm{p}_{\mathrm{p}}, \mathrm{TEE}_{\mathrm{PA}}$ energy expenditure (PAEE) and PAL using $\mathrm{RMR}_{\mathrm{p}}$, and PAL using $\mathrm{RMR}_{\mathrm{m}}$ assessed by the DLW method and accelerometry. The present data showed that while accelerometry estimated TEE accurately, it did not provide the precise measurement of PAEE and PAL. The error in accelerometry was attributed to the prediction error of RMR and assessment in exercise.

Key words: Junior high school students: Total energy expenditure: Walking step counts: Sports

Data from the Ministry of Education, Culture, Sports, Science, and Technology in Japan show that the proportion of obese (more than $120 \%$ of standard body weight for height) Japanese junior high school students (aged 12-15 years old) is $9 \cdot 37-10.99 \%$ in boys and $7 \cdot 89-8.92 \%$ in girls, and that the proportion in boys has increased slightly over the last 20 years $^{(1)}$. A change in physical activity (PA) may have effected this increase in the proportion of obesity, although data on PA in Japanese adolescents are limited.

The proportion of junior high school students participating in daily exercise increased from 1970 to $2000^{(2)}$. However, the PA level (PAL) and the amount of moderate-to-vigorous PA (MVPA) were not established in previous studies.

Studies of PA in Japanese adolescents often measured walking steps, as pedometers and accelerometers are very popular tools in Japan. These studies used uniaxial accelerometry to show that junior high school students walk 9450-15428 steps/d on weekdays and 6375-15517 steps/d on weekends ${ }^{(3)}$.
Only two studies have measured total PA using the doubly labelled water (DLW) method in Japanese children and adolescents. Hikihara et al. ${ }^{(4)}$ measured PA in high school baseball players (mean age 16.5 years, PAL 2.66), while Adachi et al. ${ }^{(5)}$ measured PA in elementary school students (mean age 11.2 years, PAL 1.47). These studies simultaneously used uniaxial accelerometry, which is used widely in Japan. Hikihara et al. ${ }^{(4)}$ reported that total energy expenditure (TEE) measured by uniaxial accelerometry correlated strongly with DLW data $(r 0.73, P<0.05)$, but underestimated TEE (-35.3 (SD 3.6)\%).

Subjective measurements are less preferable in children as a consequence of complex movement behaviour in young people and their inability to accurately recall the intensity, frequency and duration of activities ${ }^{(6)}$. Objective measurements using accelerometers are therefore required to evaluate $\mathrm{PA}^{(7,8)}$. Possible reasons for the limited data on PAL and MVPA in Japanese children are the lack of a validation

Abbreviations: ACC $_{\mathrm{m}}$, measured by accelerometry using measured RMR; ACC $\mathrm{p}$, measured by accelerometry using predicted RMR; DLW, doubly labelled

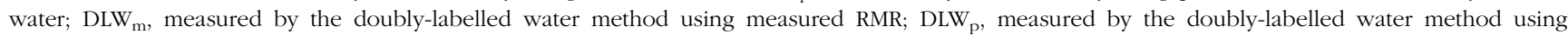
predicted RMR; TEE, total energy expenditure; MVPA, moderate-to-vigorous physical activity; PA, physical activity; PAEE, physical activity energy expenditure; PAL, physical activity level; $r_{\mathrm{CO}_{2}}$, rate of $\mathrm{CO}_{2}$ production; $\mathrm{RMR}_{\mathrm{m}}$, measured RMR; RMR $\mathrm{p}$, predicted RMR; TEE, total energy expenditure.

*Corresponding author: K. Ishikawa-Takata, email kazu@nih.go.jp 
study for accelerometry in children, and the higher cost of accelerometers compared with pedometers. Recently, Yamada et $a l .{ }^{(9)}$ found that a commercially available triaxial accelerometer (EW4800P; Panasonic Corporation) had relatively high accuracy for measuring PA in elderly Japanese subjects.

The objective of the present study was to evaluate the accuracy of EW4800P triaxial accelerometry for measuring PA in Japanese adolescents. This was achieved by comparing EW4800P data with PA measured by the DLW method.

\section{Experimental methods}

\section{Participants}

Subjects were recruited from one junior high school in Kanagawa Prefecture, near the Tokyo metropolitan area of Japan. Information on the study was sent to all students ( $n$ 300), and the purpose, methods and risks of the study were explained to students and their parents. No exclusion criteria were used in the recruitment of the subjects. A total of eighty students and their parents submitted written informed consent. Due to incorrect urine sampling, two subjects (one boy and one girl) were not included in the calculation of TEE by the DLW method. In addition, one subject was excluded, as she had a fever during the experiment. The periods when the accelerometer device was not worn were assessed from the accelerometer data and records kept by the students when the device was attached or removed. Valid days were defined as a non-wear time of less than five waking hours/d. In order for data to be used in the study, subjects had to wear the accelerometer for more than four weekdays and one weekend day. Of the subjects, nineteen were excluded because of faulty accelerometry data (for the two subjects described above, TEE data measured by both DLW and accelerometry were faulty). The remaining sixty subjects were included in the final analysis of the present study. The study was conducted according to the guidelines of the Declaration of Helsinki, and all procedures involving human subjects were approved by the Ethics Committee of Yokohama National University (three of the authors attended this university when the study was conducted).

\section{Study schedule}

Before the study period, anthropometric measurements, baseline urine sampling, dietary assessment and measurement of RMR $\left(\mathrm{RMR}_{\mathrm{m}}\right)$ were performed. TEE was then measured over 1 week by the DLW method ( $\mathrm{TEE}_{\mathrm{DLW}}$ ), with simultaneous collection of triaxial accelerometer data and completion of a simple PA record. The measurements were carried out from September 2006 to January 2007 depending on each student's schedule. Long vacations and periods of unusual PA, such as examination periods and special school events, were excluded from the experimental period.

\section{Doubly labelled water method}

After providing a baseline urine sample, a single dose of approximately $0.06 \mathrm{~g} / \mathrm{kg}$ body weight ${ }^{2} \mathrm{H}_{2} \mathrm{O}(99.8$ atom $\%$; Cambridge Isotope Laboratories) and $1.4 \mathrm{~g} / \mathrm{kg}$ body weight $\mathrm{H}_{2}{ }^{18} \mathrm{O}$ (10.0 atom\%; Taiyo Nippon Sanso), was administered orally to each subject. Subjects were then asked to collect urine samples immediately after arriving at school at the same time every morning over 1 week. On Saturday and Sunday, subjects collected urine samples at home at the same time as on school days. All samples were stored frozen at $-30^{\circ} \mathrm{C}$ in airtight parafilm-wrapped containers until analysis in our laboratory.

Gas samples for the isotope ratio mass spectrometer were prepared by equilibration of the urine sample with a gas. The gas used for equilibration of ${ }^{18} \mathrm{O}$ was $\mathrm{CO}_{2}$ and $\mathrm{H}_{2}$ was used for ${ }^{2} \mathrm{H}$ equilibration. A Pt catalyst (R1091830; Thermo Electron Corporation) was also used for the equilibration of ${ }^{2} \mathrm{H}$. The urine samples were analysed in a DELTA Plus isotope ratio mass spectrometer (Thermo Electron Corporation). Each sample and the corresponding reference sample were analysed in duplicate. Throughout the analysis, average standard deviations were $0.5 \%$ for ${ }^{2} \mathrm{H}$ and $0.03 \%$ for ${ }^{18} \mathrm{O}$.

The ${ }^{2} \mathrm{H}$ and ${ }^{18} \mathrm{O}$ zero-time intercepts and elimination rates $\left(k_{\mathrm{H}}\right.$ and $k_{\mathrm{O}}$ ) were calculated using least-squares linear regression of the natural logarithm of isotope concentration as a function of the time elapsed since dose administration. Zero-time intercepts were used to determine isotope pool sizes. The total body water was calculated as the mean value of the isotope pool size of ${ }^{2} \mathrm{H}\left(N_{\mathrm{d}}\right)$ divided by 1.041 and that of ${ }^{18} \mathrm{O}\left(N_{\mathrm{O}}\right)$ divided by 1.007 . In the present study, $N_{\mathrm{d}} / N_{\mathrm{O}}$ was 1.021 (SD 0.012 ) (range $1.000-1.055$ ), which is within the recommended range for high-quality analysis ${ }^{(10)}$. The rate of $\mathrm{CO}_{2}$ production $\left(r_{\mathrm{CO} 2}\right)$ was calculated as:

$$
r_{\mathrm{CO}_{2}}=0.4554 \times \mathrm{TBW} \times\left(1.007 k_{\mathrm{O}}-1.041 k_{\mathrm{H}}\right),
$$

where TBW is the total body water, and we assumed that isotope fractionation applied to both breath and transdermal water using equation (A6) from Schoeller et al. ${ }^{(11)}$ with the revised dilution space constant of Racette et al. ${ }^{(12)}$. Calculation of $\mathrm{TEE}_{\mathrm{DLW}}(\mathrm{kJ} / \mathrm{d})$ was performed using a modification of Weir's formula based on the $r_{\mathrm{CO} 2}$ and mean food quotient of the subjects in the study ${ }^{(13,14)}$. The food quotient was calculated based on dietary data from a brief self-administered dietary history questionnaire for junior high and high school students $^{(15)}$. The intake of protein, fat and carbohydrate assessed from this questionnaire correlated with data obtained from dietary records, with Pearson's correlation coefficients ranging from 0.38 to $0.68^{(16)}$. The mean food quotient of subjects in the present study was 0.87 (SD 0.02), which is very similar to the values of 0.87 (SD 0.03 ) for Japanese adults ${ }^{(17)}$ and 0.86 (SD 0.04) for elderly Japanese subjects ${ }^{(9)}$. TEE DLW was expressed as mean $\mathrm{TEE}_{\mathrm{DLw}}$ per $\mathrm{d}$ over the study period. However, we did not adjust $\mathrm{TEE}_{\mathrm{DLw}}$ for the time at which the accelerometer was not worn, as the non-wear time was minimal in all the study subjects. PA energy expenditure (PAEE) was calculated as $\left(\mathrm{TEE}_{\mathrm{DLW}} \times 0.9-\mathrm{RMR}\right)$ using both $\mathrm{RMR}_{\mathrm{m}}\left(\mathrm{PAEE}_{\mathrm{DLWm}}\right)$ and predicted RMR $\left(\mathrm{RMR}_{\mathrm{p}}, \mathrm{PAEE}_{\mathrm{DLW}_{\mathrm{p}}}\right)$, 
assuming that dietary-induced thermogenesis accounted for $10 \%$ of $\mathrm{TEE}_{\mathrm{DLW}}$. PAL was calculated as $\mathrm{TEE}_{\mathrm{DLW}}$ divided by $\mathrm{RMR}_{\mathrm{m}}$ or $\mathrm{RMR}_{\mathrm{p}}\left(\mathrm{PAL}_{\mathrm{DLWm}}, \mathrm{PAL}_{\mathrm{DLW}}\right)$. Fat-free mass was calculated as the proportion of water in fat-free mass and was determined to be $74.5 \%$ for boys and $75.5 \%$ for girls ${ }^{(18)}$.

\section{Triaxial accelerometry}

A triaxial accelerometer (EW4800P; Panasonic Corporation) was secured at the waist by a rubber belt throughout the period of DLW measurements. Details on the accelerometer and its accuracy in elderly Japanese have been reported in a study by Yamada et al. ${ }^{(9)}$. The accelerometer measures $60 \times 35 \times 13 \mathrm{~mm}$ and weighs $24 \mathrm{~g}$. It has a linear frequency response with a low-pass filter, and samples acceleration at $20 \mathrm{~Hz}$ with a range from zero to two times the acceleration of gravity. The device stores the standard deviation of the vector norm of the composite acceleration $(\mathrm{Km})$ in three dimensions each minute as follows:

where $n$ is the number of data for $1 \min (n 1200)$, and $\Sigma x, \Sigma y$, and $\Sigma z$ are the sums of the acceleration in three directions over $1 \mathrm{~min}$. Data were not rounded off for storage of $\mathrm{Km}$.

Subjects were asked to wear the accelerometer for the whole day except when sleeping, bathing or swimming. If subjects took the accelerometer off due to difficulty in wearing it during contact sports, or if they forgot to attach it, they were asked to record the time of removal and the duration and type of PA carried out while not wearing the accelerometer. A commercially available accelerometry software (EW48001; Panasonic Corporation) was used to calculate the total energy expenditure measured by the accelerometer using $\mathrm{RMR}_{\mathrm{p}}\left(\mathrm{TEE}_{\mathrm{ACCP}}\right)$, walking steps and duration of light PA (less than 3 metabolic equivalents), moderate PA (3-6 metabolic equivalents) and vigorous PA (6 metabolic equivalents or more). The metabolic equivalent intensity levels of physical activities were calculated using the simple linear regression of $\mathrm{Km}$ and $\mathrm{O}_{2}$ consumption ${ }^{(19)}$. MVPA was calculated as the sum of moderate PA and vigorous PA. The commercially available software incorporated $\mathrm{RMR}_{\mathrm{p}}$ calculated using standard values to calculate $\mathrm{TEE}_{\mathrm{ACCp}}$ that were obtained for the Japanese population from the dietary reference intake in Japan ${ }^{(20)}$. PAEE assessed by accelerometry ( PAEE $_{\mathrm{ACCP}}$ ) was calculated as $\mathrm{TEE}_{\mathrm{ACCP}} \times 0.9-\mathrm{RMR}_{\mathrm{p}}$. $\mathrm{PAL}_{\mathrm{ACCP}}$ was calculated as $\mathrm{TEE}_{\mathrm{ACCP}} /$ $\mathrm{RMR}_{\mathrm{p}}$. In the present study, we also calculated each value using raw accelerometry data and $\mathrm{RMR}_{\mathrm{m}}$ by the same equations used in the commercially available software $\left(\mathrm{TEE}_{\mathrm{ACCm}}, \mathrm{PAEE}_{\mathrm{ACCm}}\right.$ and $\left.\mathrm{PAL}_{\mathrm{ACCm}}\right)$.

\section{$R M R$}

Before the study period, subjects were asked to come to school at 08.00 hours after an overnight fast. After 30 min of rest, $\mathrm{RMR}_{\mathrm{m}}$ was measured using an indirect calorimeter with a hood (AR-1; Arco System) at an environmental temperature between 22 and $25^{\circ} \mathrm{C}$. This system involved a steady state being established 3 min after the starting of collecting respiratory gas, with the accuracy of RMR measured by this system being $0.02 \%{ }^{(21)}$. Subjects were placed under a transparent plastic hood that covered their heads and that was connected to the system. After the child had adapted to the hood for $5 \mathrm{~min}$, RMR was measured for $20 \mathrm{~min} . \mathrm{O}_{2}$ consumption and $\mathrm{CO}_{2}$ production were measured at $1 \mathrm{~min}$ intervals and averaged over 6-20 min. Resting status was confirmed by measuring the body temperature and the heart rate.

Table 1. Anthropometric characteristics of the study subjects

(Mean values and standard deviations)

\begin{tabular}{|c|c|c|c|c|c|c|c|c|c|c|c|c|c|c|c|}
\hline & \multirow[b]{2}{*}{$n$} & \multicolumn{2}{|c|}{ Age (years) } & \multicolumn{2}{|c|}{ Height $(\mathrm{cm})$} & \multicolumn{2}{|c|}{ Weight (kg) } & \multicolumn{2}{|c|}{ Body fat (\%) } & \multicolumn{2}{|c|}{$\begin{array}{l}\text { TEE }_{D L W} \\
(\mathrm{MJ} / \mathrm{d})\end{array}$} & \multicolumn{2}{|c|}{$\begin{array}{l}\mathrm{RMR}_{\mathrm{m}} \\
(\mathrm{MJ} / \mathrm{d})\end{array}$} & \multicolumn{2}{|c|}{$P A L_{D L W m}$} \\
\hline & & Mean & $\mathrm{SD}$ & Mean & SD & Mean & SD & Mean & $\mathrm{SD}$ & Mean & $\mathrm{SD}$ & Mean & SD & Mean & SD \\
\hline Boys & 12 & $12 \cdot 7$ & 0.7 & $158 \cdot 3$ & $10 \cdot 7$ & $48 \cdot 1$ & $10 \cdot 7$ & $22 \cdot 6$ & $6 \cdot 1$ & 11.9 & 2.9 & 5.9 & 1.0 & 1.98 & 0.26 \\
\hline Girls & 7 & $12 \cdot 1$ & 0.4 & $152 \cdot 9$ & 7.5 & $40 \cdot 6$ & 8.4 & $24 \cdot 6$ & 6.9 & $9 \cdot 1$ & 1.3 & $4 \cdot 8$ & 0.5 & 1.93 & 0.29 \\
\hline \multicolumn{16}{|l|}{ Grade 2} \\
\hline \multicolumn{16}{|l|}{ Grade 3} \\
\hline Boys & 10 & $14 \cdot 6$ & 0.8 & $163 \cdot 3$ & $9 \cdot 4$ & $52 \cdot 4$ & 4.4 & $17 \cdot 0$ & 4.4 & $11 \cdot 7$ & 1.9 & $6 \cdot 4$ & 0.5 & 1.83 & 0.32 \\
\hline Girls & 9 & $14 \cdot 0$ & 0.0 & $156 \cdot 1$ & $5 \cdot 9$ & $46 \cdot 1$ & 4.5 & 24.9 & $4 \cdot 6$ & $9 \cdot 8$ & $2 \cdot 0$ & $5 \cdot 2$ & 0.3 & 1.87 & 0.32 \\
\hline \multicolumn{16}{|l|}{$P$} \\
\hline Grade & & & & 0.003 & & 0.098 & & 0.027 & & 0.331 & & 0.037 & & 0.472 & \\
\hline Sex & & & & $<0.001$ & & $<0.001$ & & $<0.001$ & & $<0.001$ & & $<0.001$ & & 0.142 & \\
\hline Grade $\times$ sex & & & & 0.708 & & 0.900 & & 0.096 & & 0.111 & & 0.777 & & 0.115 & \\
\hline
\end{tabular}

$\mathrm{TEE}_{\mathrm{DLW}}$, total energy expenditure measured by the doubly labelled water method; $\mathrm{RMR}_{\mathrm{m}}$, measured RMR; PAL $\mathrm{DLWm}_{\mathrm{m}}$, physical activity level measured by the doubly labelled water method. 
Table 2. Total and physical activity energy expenditure of junior high school students who did and did not exercise

(Mean values and standard deviations; medians and 25th-75th percentiles)

\begin{tabular}{|c|c|c|c|c|c|c|c|c|c|c|c|c|c|c|c|c|c|c|}
\hline & & & & & & & & & & & & & & & \multicolumn{4}{|c|}{ Non-wear time $(\mathrm{min} / \mathrm{d})^{\star}$} \\
\hline & \multicolumn{2}{|c|}{$\begin{array}{l}\text { Percentage } \\
\text { of body fat }\end{array}$} & \multicolumn{2}{|c|}{$\begin{array}{l}T_{E E_{D L W}} \\
(\mathrm{MJ} / \mathrm{d})\end{array}$} & \multicolumn{2}{|c|}{$\mathrm{RMR}_{\mathrm{m}}(\mathrm{MJ} / \mathrm{d})$} & \multicolumn{2}{|c|}{$\begin{array}{l}\text { PAEE }_{\text {DLWm }} \\
(\mathrm{MJ} / \mathrm{d})\end{array}$} & \multicolumn{2}{|c|}{$P A L_{D L W m}$} & \multicolumn{2}{|c|}{$\begin{array}{l}\text { TEE }_{A C C p} \\
(\mathrm{MJ} / \mathrm{d})\end{array}$} & \multicolumn{2}{|c|}{$\begin{array}{l}\text { Walking steps } \\
\text { (steps/d) }\end{array}$} & \multicolumn{2}{|c|}{ Weekday } & \multicolumn{2}{|c|}{ Weekend } \\
\hline & Mean & SD & Mean & SD & Mean & SD & Mean & SD & Mean & SD & Mean & SD & Mean & SD & Mean & SD & Mean & $\mathrm{SD}$ \\
\hline \multicolumn{19}{|l|}{ Boys } \\
\hline $\operatorname{Ex}(n 27) \dagger$ & $17 \cdot 3$ & $8 \cdot 1$ & $12 \cdot 7$ & $2 \cdot 4$ & $6 \cdot 3$ & 0.8 & $5 \cdot 2$ & 1.7 & $2 \cdot 03$ & 0.30 & $12 \cdot 1$ & $2 \cdot 0$ & 15920 & 3730 & 57 & 40 & 77 & 82 \\
\hline Median & & & \multirow{2}{*}{\multicolumn{2}{|c|}{$\begin{array}{c}12 \cdot 9 \\
10 \cdot 9-14 \cdot 1\end{array}$}} & \multirow{2}{*}{\multicolumn{2}{|c|}{$\begin{array}{c}6 \cdot 4 \\
5 \cdot 8-6 \cdot 7\end{array}$}} & \multirow{2}{*}{\multicolumn{2}{|c|}{$\begin{array}{c}5.5 \\
3.8-6.7\end{array}$}} & \multirow{2}{*}{\multicolumn{2}{|c|}{$\begin{array}{c}2 \cdot 03 \\
1.78-2 \cdot 2\end{array}$}} & \multirow{2}{*}{\multicolumn{2}{|c|}{$\begin{array}{c}12 \cdot 0 \\
10 \cdot 8-13 \cdot 7\end{array}$}} & \multirow{2}{*}{\multicolumn{2}{|c|}{16553}} & & & & \\
\hline 25th-75th percentile & & & & & & & & & & & & & & & & & & \\
\hline Non-Ex ( $n 5) \ddagger$ & $21 \cdot 6$ & 4.7 & 11.0 & $2 \cdot 4$ & $6 \cdot 5$ & $1 \cdot 0$ & $3 \cdot 4$ & 1.4 & 1.69 & 0.20 & $10 \cdot 8$ & $1 \cdot 2$ & 11170 & 3182 & 65 & 30 & 70 & 69 \\
\hline Median & & & \multirow{2}{*}{\multicolumn{2}{|c|}{$\begin{array}{c}11 \cdot 1 \\
8 \cdot 9-13 \cdot 0\end{array}$}} & \multirow{2}{*}{\multicolumn{2}{|c|}{$\begin{array}{c}6 \cdot 8 \\
5.6-7.1\end{array}$}} & \multirow{2}{*}{\multicolumn{2}{|c|}{$\begin{array}{c}3.5 \\
2 \cdot 1-4 \cdot 7\end{array}$}} & \multirow{2}{*}{\multicolumn{2}{|c|}{$\begin{array}{c}1.71 \\
1.51-1.86\end{array}$}} & \multirow{2}{*}{\multicolumn{2}{|c|}{$\begin{array}{c}11 \cdot 2 \\
9 \cdot 2-12 \cdot 3\end{array}$}} & \multirow{2}{*}{\multicolumn{2}{|c|}{$\begin{array}{c}10893 \\
8378-14100\end{array}$}} & & & & \\
\hline 25th-75th percentile & & & & & & & & & & & & & & & & & & \\
\hline Total $(n$ 32) & $18 \cdot 0$ & $7 \cdot 7$ & 12.4 & $2 \cdot 4$ & $6 \cdot 3$ & 0.8 & 4.9 & 1.8 & 1.97 & 0.31 & 11.9 & $2 \cdot 0$ & 15178 & 4006 & 58 & 38 & 76 & 79 \\
\hline Median & & & \multirow{2}{*}{\multicolumn{2}{|c|}{$\begin{array}{c}12 \cdot 6 \\
10 \cdot 7-14 \cdot 0\end{array}$}} & \multirow{2}{*}{\multicolumn{2}{|c|}{$\begin{array}{c}6 \cdot 5 \\
5 \cdot 8-6 \cdot 8\end{array}$}} & \multirow{2}{*}{\multicolumn{2}{|c|}{$\begin{array}{c}5 \cdot 1 \\
3 \cdot 2-6 \cdot 4\end{array}$}} & & & & & 15 & & & & & \\
\hline 25th-75th percentile & & & & & & & & & 1.73 & 2.16 & $10 \cdot($ & 3.4 & 13046 & 7437 & & & & \\
\hline Girls & & & & & & & & & & & & & & & & & & \\
\hline $\operatorname{Ex}(n 18) \dagger$ & $22 \cdot 8$ & 2.5 & $9 \cdot 7$ & $1 \cdot 7$ & $5 \cdot 1$ & 0.4 & $3 \cdot 6$ & 1.4 & 1.90 & 0.30 & $9 \cdot 3$ & $1 \cdot 1$ & 13289 & 2365 & 72 & 55 & 101 & 78 \\
\hline Median & & & & & & & & & & & & & 13 & & & & & \\
\hline 25th-75th percentile & & & $8 \cdot 4$ & & & & & & 1.73 & 2.06 & & & 12047 & 4503 & & & & \\
\hline Non-Ex ( $n$ 10)‡ & $26 \cdot 7$ & $6 \cdot 0$ & $8 \cdot 7$ & $1 \cdot 2$ & $5 \cdot 0$ & 0.4 & $2 \cdot 9$ & 1.0 & $1 \cdot 75$ & 0.21 & $9 \cdot 4$ & $1 \cdot 6$ & 12303 & 2001 & 69 & 29 & 70 & 40 \\
\hline Median & & & & & & & & & & & & & 12 & & & & & \\
\hline 25th-75th percentile & & & 7.9 & & & & & & 1.57 & 1.91 & & & 10869 & 3381 & & & & \\
\hline Total $(n$ 28) & $24 \cdot 2$ & 4.4 & $9 \cdot 3$ & $1 \cdot 6$ & $5 \cdot 1$ & 0.4 & $3 \cdot 3$ & $1 \cdot 3$ & 1.85 & 0.27 & $9 \cdot 3$ & $1 \cdot 3$ & 12937 & 2256 & 71 & 47 & 90 & 68 \\
\hline Median & & & $\varepsilon$ & & & & & & & & & & 12 & & & & & \\
\hline 25th-75th percentile & & & $8 \cdot 2$ & & 4.8 & & $2 \cdot 5$ & & 1.67 & 1.94 & & & 11974 & 4131 & & & & \\
\hline$P$ & & & & & & & & & & & & & & & & & & \\
\hline Exercise & 0.0 & & & & & & & & & & & & 0.6 & & $0 \cdot 8$ & & 0.4 & \\
\hline Sex & $0 \cdot 0$ & & $<0$ & & & & & & & & & & 0.4 & & 0.4 & & 0.6 & \\
\hline Exercise $\times$ sex & 0.9 & & & & & & & & & & & & $0 . c$ & & 0.6 & & 0.6 & \\
\hline
\end{tabular}

$T_{E E_{D L W}}$, total energy expenditure measured by the doubly labelled water method; $R_{M} R_{m}$, measured RMR; PAEE $E_{D L W m}$, physical activity energy expenditure $\left(T E E_{D L W} \times 0.9-R_{M M R}\right) ; P A L_{D L W m}$, physical activity level measured by the doubly labelled water method using $\mathrm{RMR}_{\mathrm{m}}\left(\mathrm{TEE}_{\mathrm{DLw}} / \mathrm{RMR}_{\mathrm{m}}\right) ; \mathrm{TEE}_{\mathrm{ACCp}}$, total energy expenditure measured by the accelerometer using predicted RMR.

*Non-wear time: duration of the time that subjects did not wear the accelerometer.

Fx: subjects who exercised except for the physical education class.

‡Non-Ex: subjects who did not exercise except for the physical education class. 


\section{Statistical analysis}

All the analyses were performed using SPSS $16.0 \mathrm{~J}$ for Windows (IBM Japan). The characteristics of the subjects are expressed as means and standard deviations. As the PA data were not normally distributed, they were expressed as both means and standard deviations and medians with ranges. Differences between grades and sexes were analysed by two-way ANOVA. The linear relationships between PAL and light PA, moderate PA, vigorous PA and MVPA were assessed using Pearson's correlation coefficients. Student's paired $t$ test, Spearman's rank correlation coefficients, intraclass correlation coefficient and Bland-Altman plot analysis ${ }^{(22)}$ were used to compare data obtained from the DLW method and accelerometry.

\section{Results}

The physical characteristics of the subjects are shown in Table 1. Height and weight were significantly lower in girls than in boys (both $P<0.001$ ). The percentage of body fat was higher in girls than in boys $(P<0 \cdot 001)$. TEE $E_{\text {DLW }}$ and $\mathrm{RMR}_{\mathrm{m}}$ were significantly lower in girls than in boys. $\mathrm{PAL}_{\mathrm{DLW}}$ ranged from 1.48 to 2.54 in individual subjects, with a mean of 1.91. PAL $_{\text {DLWm }}$ was not different between sexes or grades. All subjects included in the analysis wore the accelerometers for more than four weekdays and more than one weekend day. Of the subjects, $82 \%$ wore the accelerometer for five weekdays, and $90 \cdot 2 \%$ wore it for two weekend days. TEE DLW $_{\text {and }}$ PAL $_{\text {DLWm }}$ were not different between the subjects included in the analysis and subjects excluded due to insufficient accelerometer use (TEE DLW $_{11.0}(\mathrm{SD} 2 \cdot 6) v .11 \cdot 0(\mathrm{SD} 2 \cdot 5) \mathrm{MJ} / \mathrm{d}$; $\begin{array}{llllllll}\mathrm{RMR}_{\mathrm{m}} & 5.7(\mathrm{SD} & 0.9) & v & 5.9 & (\mathrm{SD} & 1.1) \mathrm{MJ} / \mathrm{d} ; \mathrm{PAL}_{\mathrm{DLWm}} & 1.91\end{array}$ (SD 0.30) v.1.85 (SD 0.26), respectively).

Of the present subjects, $75 \%$ (twenty-seven boys and ten girls) participated in exercise other than the physical education class. The frequency and duration of exercise were 3.6 (SD 1.8) times/week and 523 (SD 291) min/week for boys, and 2.1 (SD 1.1) times/week and 318 (SD 219) min/week for girls, respectively. Major sports activities included kendo ( $n$ 5), soccer $(n 4)$, tennis ( $n$ 4), basketball $(n 4)$, track and field ( $n 4)$ and baseball ( $n$ 3) for boys, and badminton $(n 6)$, track and field $(n 5)$ and tennis $(n 3)$ for girls. The percentage of body fat was significantly lower in subjects who exercised; however, $\mathrm{RMR}_{\mathrm{m}}$ did not differ with exercise activity (Table 2). Non-wear time, including bathing, periods of PA preventing accelerometer use and times when participants forgot to wear the accelerometer, also did not differ with exercise activity. $\mathrm{TEE}_{\mathrm{DLW}}, \mathrm{TEE}_{\mathrm{ACCm}}$ and $\mathrm{TEE}_{\mathrm{ACCp}}$ were $11 \cdot 0$ (SD 2.0), $10 \cdot 3$ (SD 1.9) and $10 \cdot 7$ (SD 2.1) MJ/d for all subjects, respectively. All three indices were significantly greater in subjects who exercised compared with subjects who did not exercise. Walking step counts were greater in subjects who exercised; however, $\mathrm{TEE}_{\mathrm{ACCP}}$ did not differ with exercise activity.

$\mathrm{RMR}_{\mathrm{m}}$ and $\mathrm{RMR}_{\mathrm{p}}$ correlated strongly in all subjects and in subjects who exercised, although $\mathrm{RMR}_{\mathrm{p}}$ was significantly greater than $\mathrm{RMR}_{\mathrm{m}}$ in all subjects and subjects who did not exercise (Fig. 1; Table 3). The percentage difference between

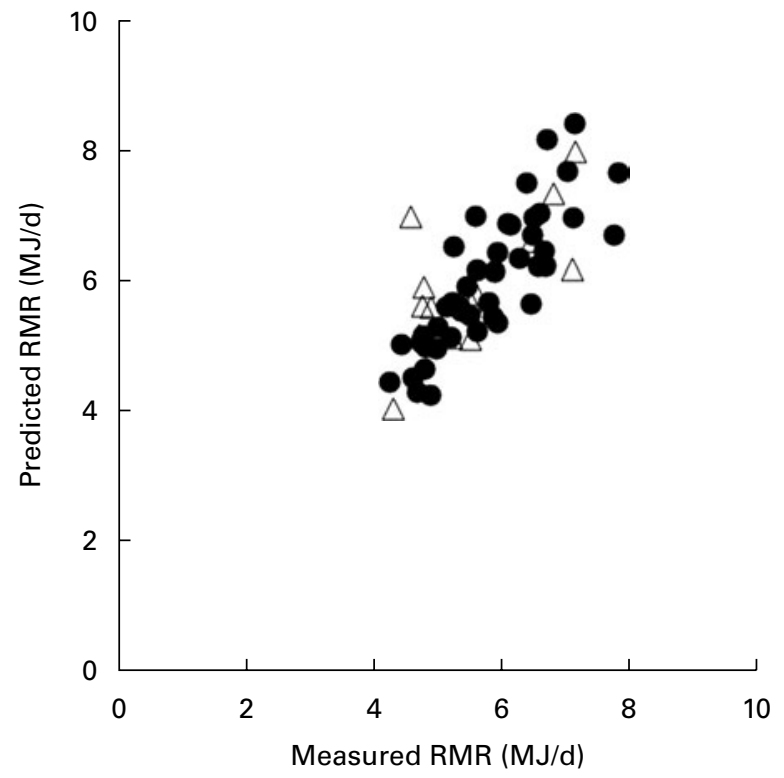

Fig. 1. Correlation between measured and predicted RMR. $\bullet$, Subjects who exercised; $\Delta$, subjects who did not exercise. The Spearman's correlation coefficients were $0.732(P<0.001)$ for all subjects, $0.800(P<0.001)$ for subjects who exercised, and $0.436(P=0.020)$ for subjects who did not exercise.

$\mathrm{RMR}_{\mathrm{m}}$ and $\mathrm{RMR}_{\mathrm{p}}$ correlated with body weight ( $r$ 0.401, $P=0.002)$ and the percentage of body fat $(r 0.524, P<0.001)$, but not with sex, age or height. $\mathrm{TEE}_{\mathrm{DLW}}$ correlated significantly with both $\mathrm{TEE}_{\mathrm{ACC}}$ and $\mathrm{TEE}_{\mathrm{ACCp}}$, although $\mathrm{TEE}_{\mathrm{ACCp}}$ was significantly smaller than $\mathrm{TEE}_{\mathrm{DLW}}$ in all the subjects. In subjects who exercised, accelerometry underestimated TEE significantly using either $\mathrm{RMR}_{\mathrm{m}}$ or $\mathrm{RMR}_{\mathrm{p}}$. Spearman's rank correlation coefficients and intraclass correlation coefficient were lower for PAEE than for TEE in all subjects and in the exercising and non-exercising subjects. Accelerometry underestimated PAEE significantly in comparisons between $\operatorname{PAEE}_{\mathrm{DLW}} v \cdot \mathrm{PAEE}_{\mathrm{ACCm}}$, and $\mathrm{PAEE}_{\mathrm{DLW}} v \cdot \mathrm{PAEE}_{\mathrm{ACCP}}$ in all subjects and in subjects who exercised. Subjects who did not exercise showed a weaker correlation between PAEE assessed by the DLW method and accelerometry. In comparison with PAL, Spearman's rank correlation coefficient and intraclass correlation coefficient were lower than those for PAEE and TEE. Accelerometry underestimated PAL only when $\mathrm{PAL}_{\mathrm{ACCP}}$ was compared with $\mathrm{PAL}_{\mathrm{DLWm}}$ in all subjects and in exercising and non-exercising subjects. The percentage of body fat correlated significantly with the percentage difference in TEE, PAEE and PAL assessed by the DLW method and accelerometry using $\mathrm{RMR}_{\mathrm{p}}(r 0.304, P=0.018$ for TEE and PAL; $r 0.349, P=0.006$ for PAEE), and PAL using $\mathrm{RMR}_{\mathrm{m}}(r$ 0.304, $P=0 \cdot 018)$. Sex, age, body weight and height did not correlate significantly with the percentage difference between TEE, PAEE and PAL. The Bland-Altman agreement plots showed a moderate negative correlation between PAL assessed by either the DLW method or accelerometry, even when measured or $\mathrm{RMR}_{\mathrm{p}}$ was used (Fig. 2).

When the subjects were divided into tertile subgroups for PAL, 47, 45 and $52 \%$ of the subjects were stratified into the same tertiles of $\mathrm{PAL}_{\mathrm{DLWm}} v$. $\mathrm{PAL}_{\mathrm{ACCm}}, \mathrm{PAL}_{\mathrm{DLW}} v$. $\mathrm{PAL}_{\mathrm{ACC}}$ 


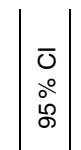

ণิ ఫ্ড

(1)

का 它全

号 ๓

$\frac{.0}{0} \frac{0}{0}$

5
0
$\frac{0}{0}$
0
$\frac{0}{3}$
$\frac{9}{0}$
$\frac{0}{0}$
$\frac{0}{3}$

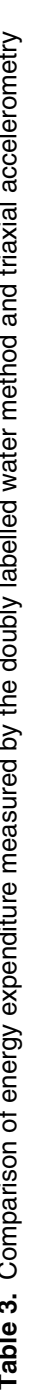

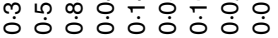

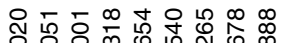

然

인 뉸

。

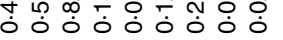

$\pm$

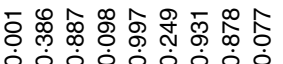

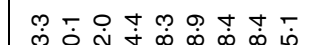

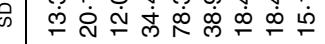

穴

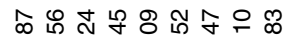

U

กั

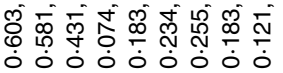

i

항

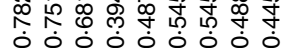

is

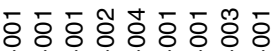

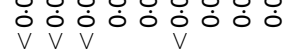

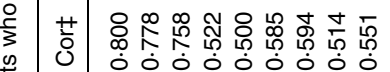

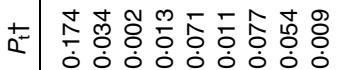

- L

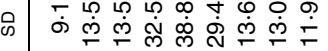

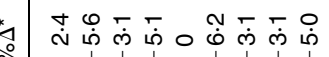

б

丁

品

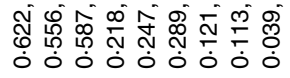

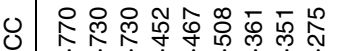

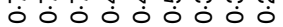

m 훙ㅎㅎㅎㅎㅎㅎㅎㅎㅎㅇㅇㅇ 웅

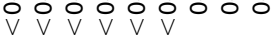

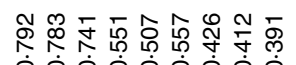

$\pm$

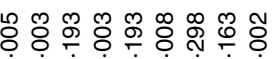

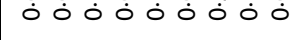

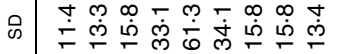

*

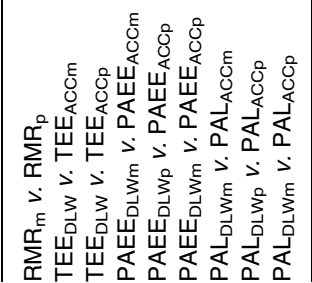

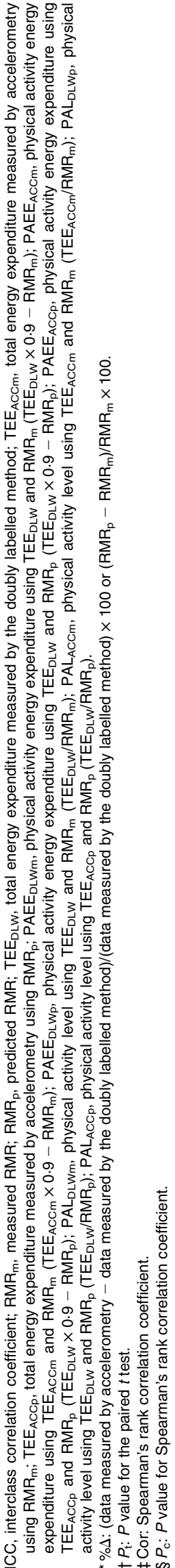

and $\mathrm{PAL}_{\mathrm{DLWm}} v$. $\mathrm{PAL}_{\mathrm{ACCP}}$. However, 27, 30 and $25 \%$ of the subjects were divided into the lower PAL subgroups according to $\mathrm{PAL}_{\mathrm{DLWm}} v$. $\mathrm{PAL}_{\mathrm{ACCm}}, \mathrm{PAL}_{\mathrm{DLW}} v$. $\mathrm{PAL}_{\mathrm{ACCP}}$ and $\mathrm{PAL}_{\mathrm{DLWm}} v$. $\mathrm{PAL}_{\mathrm{ACCP}}$.

The average number of walking steps and the duration of light PA, moderate PA, vigorous PA and MVPA assessed by accelerometry were 14132 (SD 3469) steps/d, and 894 (SD 66), 89 (SD 29), 13 (SD 14) and 103 (SD 39) $\mathrm{min} / \mathrm{d}$, respectively. MVPA in subjects who did or did not exercise was 113 (SD 38) and 72 (SD 22) min/week, respectively. MVPA in weekends was significantly shorter $(P<0.001)$ than in weekdays (81 (SD 47) v. 111 (SD 42) $\mathrm{min} / \mathrm{d})$. Walking step counts were also significantly lower $(P<0.001)$ in weekends (10630 (sD 5622) steps/d) compared with weekdays (15652 (SD 3632) steps/d). MVPA correlated significantly with $\mathrm{PAL}_{\mathrm{DLWm}}(r 0.341, P=0.008)$. The linear relationship between $\mathrm{MVPA}$ and $\mathrm{PAL}_{\mathrm{DLWm}} \quad\left(\mathrm{PAL}_{\mathrm{DLWm}}=\mathrm{MVPA} \times 0.003+1.65\right)$ showed that $30 \mathrm{~min}$ of MVPA per $\mathrm{d}$ was equivalent to 1.74 of $\mathrm{PAL}_{\mathrm{DLW}}$.

\section{Discussion}

This is the first study to assess PA among Japanese junior high school students using the DLW method and triaxial accelerometry. TEE assessed by accelerometry was found to have good accuracy as determined by comparison with $\mathrm{TEE}_{\mathrm{DLw}}$, whereas the accuracy of PAEE and PAL was lower compared with that of TEE. The errors caused by accelerometry were considered to be attributable to the error in the prediction of RMR and the assessment of exercise intensity.

The average height of the subjects in the present study was slightly higher, and the girls' height slightly lower than that in data collected in 2010 by the Ministry of Education, Culture, Sports, Science, and Technology, Japan ${ }^{(1)}$. In the present study, only three students weighed more than $120 \%$ of the standard body weight for sex, age and height in Japanese students $^{(1)}$.

The average $\mathrm{PAL}_{\mathrm{DLWm}}$ of 1.91 (SD 0.30 ) in the present study was greater than that in previous studies conducted in Western countries (PAL 1.48-1.89) ${ }^{(23-33)}$. The proportion of the present subjects performing exercise, with the exception of the physical education class, was higher than that in a previous report on the entire student population at this school (in the previous study, boys $70 \%$ and girls $46 \%)^{(34)}$. As the subjects who exercised participated in MVPA about $2 \mathrm{~h}$ each week and had a smaller percentage of body fat than non-exercising subjects, they were well trained and had higher energy expenditure.

The average number of walking steps in the present study was also greater than that in previous studies that used uniaxial accelerometry. The accuracy of step counts in the present accelerometry had already been examined in an adult population only, and it is possible that there may be differences in accuracy between adult and children ${ }^{(35)}$. The accelerometer used in the present study underestimated step counts by $18 \%$ when subjects walked $55 \mathrm{~m} / \mathrm{min}$ with a normal step frequency. However, this accelerometry did not show significant differences in step count compared with visually counted step 

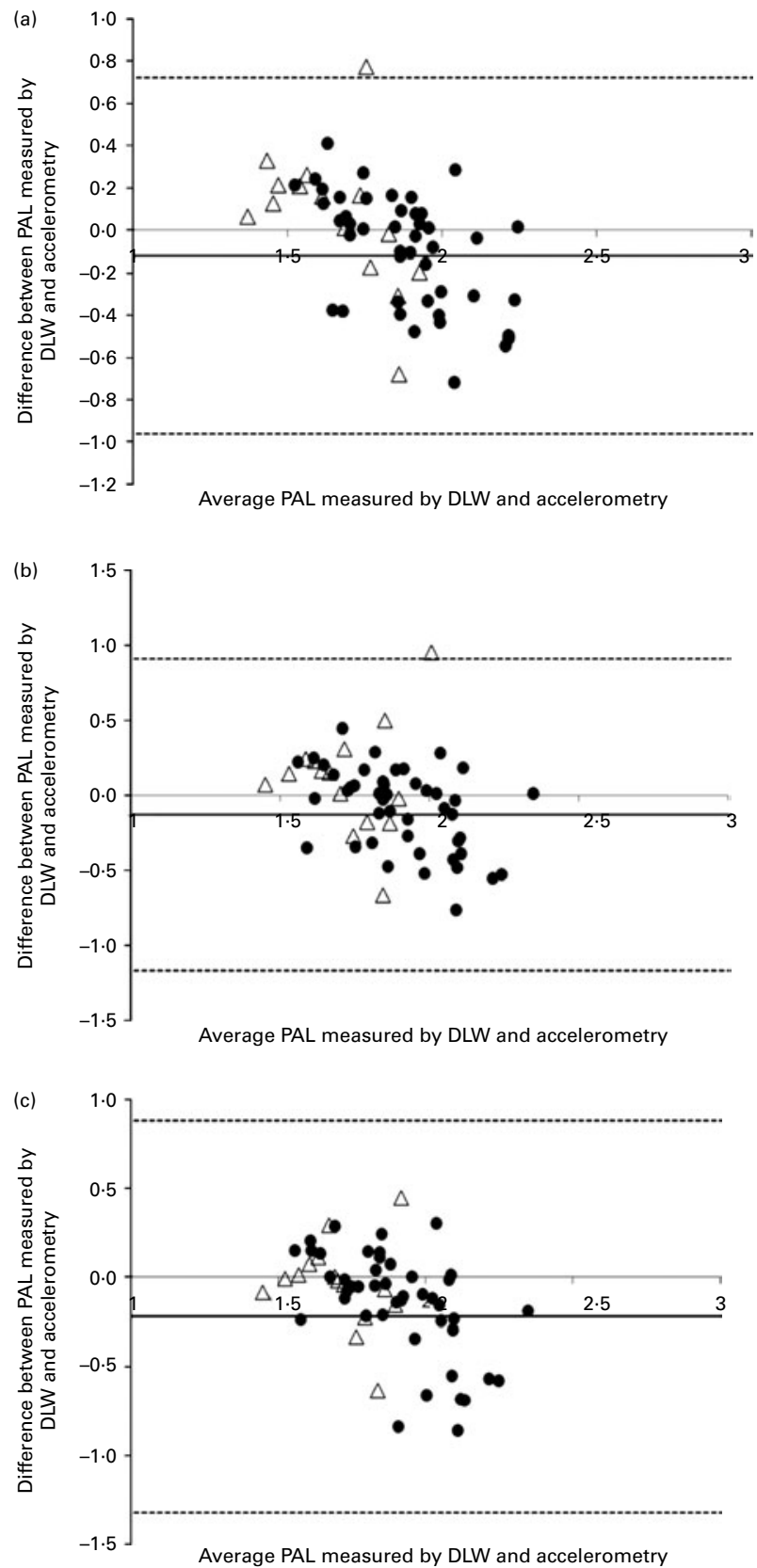

Fig. 2. Bland-Altman plots of physical activity level (PAL) assessed by either the doubly labelled water (DLW) method or an accelerometer. $\bullet$, Subjects who exercised; $\Delta$, subjects who did not exercise. - , Mean PAL measured by the DLW method and accelerometry; ---, mean (2 SD) of PAL measured by the DLW method and accelerometry. Comparison of (a) PAL measured by the DLW method and accelerometry with predicted RMR $(r-0.564, P<0.001)$, (b) PAL measured by the DLW method and accelerometry with the measured RMR $(r-0.381, P=0.003)$ and (c) PAL measured by the DLW method with the measured RMR and accelerometry with the predicted RMR $(r-0.508, P<0.001)$.

counts at walking speeds of 75 and $95 \mathrm{~m} / \mathrm{min}$. Although we could not examine the accuracy of assessing MVPA by accelerometry, the relationship between MVPA and the number of walking steps was similar to that reported in previous studies. Tudor-Locke et al $^{(36)}$ recommended 10000-11700 steps/d for adolescents to satisfy $60 \mathrm{~min}$ of MVPA. In the present study, 60 min of MVPA was equivalent to an average of 11006 steps/d.

The present study shows that TEE measured by EW4800P triaxial accelerometry had good accuracy. The percentage difference between $\mathrm{TEE}_{\mathrm{DLW}}$ and $\mathrm{TEE}_{\mathrm{ACCp}}$ was -0.7 (SD 15.8)\%. Although the commercially available software uses $\mathrm{RMR}_{\mathrm{p}}$, in the present study, we also used $\mathrm{RMR}_{\mathrm{m}}$ to calculate energy expenditure. $\mathrm{TEE}_{\mathrm{ACC}}$ showed less difference than $\mathrm{TEE}_{\mathrm{ACCm}}$ in comparison with $\mathrm{TEE}_{\mathrm{DLW}}$. The difference between $\mathrm{TEE}_{\mathrm{DLW}}$ and $\mathrm{TEE}_{\mathrm{ACCp}}$ was very close to the results of a previous study that used the same accelerometer in elderly subjects, and showed a $1.6 \%$ difference $^{(9)}$. These results suggest that this accelerometer can evaluate TEE with a similar level of accuracy in both elderly and junior high school students, at least at the group level. In addition, this accelerometer has very good accuracy compared with other accelerometers. A study using triaxial accelerometry in an age group similar to that in the present study and the most accurate estimation equation $^{(37)}$ showed that the root mean square error was $40 \cdot 72 \%$ for boys and $59 \cdot 72 \%$ for girls. We examined the effects of sex, age, body weight, height and percentage of body fat on the difference between $\mathrm{TEE}_{\mathrm{DLW}}$ and $\mathrm{TEE}_{\mathrm{ACCp}}$ or $\mathrm{TEE}_{\mathrm{ACCm}}$, and found only the percentage of body fat to be correlated significantly with the percentage difference between $\mathrm{TEE}_{\mathrm{DLW}}$ and $\mathrm{TEE}_{\mathrm{ACCp}}$. As the percentage of body fat also correlated with the difference between $R_{m}$ and $\mathrm{RMR}_{\mathrm{p}}$, the prediction error in RMR for subjects with higher body fat deposition affected the estimation error of TEE using $\mathrm{RMR}_{\mathrm{p}}$

Although TEE $_{\mathrm{ACCp}}$ and $\mathrm{TEE}_{\mathrm{ACCm}}$ showed good accuracy as established by comparison with TEE $_{\text {DLw }}$, RMR accounted for a large portion of TEE in the study subjects. To lessen the contribution of RMR, we compared PAEE and PAL measurements obtained using the two methods. The accuracy for PAEE and PAL was lower than that for TEE. For PAEE, the difference between the two methods was most apparent when $\mathrm{RMR}_{\mathrm{p}}$ was used in both the DLW method and accelerometry. In particular, the mean difference in PAEE and PAL was overestimated by accelerometry in subjects who did not exercise. The reasons for this finding were that one subject who did not exercise showed a large overestimation of PAEE by accelerometry, while the estimation error of PAEE by accelerometry was relatively small in the other non-exercising subjects. In comparison with PAL, $\mathrm{PAL}_{\mathrm{DLWm}}$ and $\mathrm{PAL}_{\mathrm{ACCm}}$ showed the strongest correlation. One reason for these results is the prediction error of $R M R$. $R M R_{p}$ is based on a standard value of RMR for Japanese in the dietary reference intake for Japan. The dietary reference intake for Japan is revised every 5 years, although the standard RMR value for children was calculated using the data collected in the 1950s. When the dietary reference intake was revised in 2010 based on data measured in the 2000s, the standard value of RMR in females aged 18-29 years decreased from 98.7 to $92.5 \mathrm{~kJ} / \mathrm{d}$. It is therefore possible that $\mathrm{RMR}_{\mathrm{p}}$ may also be overestimated in adolescents. Another possible reason for the overestimation of $\mathrm{RMR}_{\mathrm{p}}$ was the systematic error of the measurement. Cooper et $a l .{ }^{(38)}$ suggested that there was a systematic error in calorimetry systems. The system used in the present study 
has been tested previously ${ }^{(21)}$. As the standard value was based on data collected more than 50 years ago, we could not examine the systematic error between the present system and systems that were used to decide the standard value of RMR. Given that RMR can vary with age, maturation, body weight and the level of PA, we considered that a better estimation of PAEE may be obtained using a more accurate measurement of $\mathrm{RMR}^{(39)}$

In the present study, subjects who exercised tended to have a greater underestimation of PAL by accelerometry than other subjects who did not exercise. RMR and non-wear time of accelerometry were not different between the exercising and non-exercising subjects, while walking step counts were greater in subjects who exercised. As most accelerometers are more sensitive for accelerations in the vertical place and less sensitive for a more complex movement ${ }^{(40)}$, the intensity of exercise may be estimated less accurately. In addition, although the prediction model of EW4800P was constructed using data from adults, the models are population specific ${ }^{(41)}$. It is therefore possible that PA in some adolescents may not have been estimated correctly.

The most significant limitation of the present study was that all the subjects were recruited from one school. Most of the students in the present study used trains to go to school, and had to walk approximately $15 \mathrm{~min}$ each way from the nearest station to the school. In Japan, most public school students go to school on foot or by a bicycle. It is therefore necessary to collect data from other schools to generalise the present results to other Japanese junior high school students.

Second, although we sent an information letter to all the students, the participation rate was quite low. We are therefore not sure whether the subjects in the study were representative of the students in this school.

Third, some subjects had issues with improper wearing of the accelerometer. According to our criteria, approximately $25 \%$ of the subjects were excluded from the analysis because of inappropriate accelerometer use. Of the seventeen subjects excluded from the analysis, fifteen did not wear the accelerometer on any weekend days. All the subjects included in the analysis wore the accelerometer more than $12 \mathrm{~h} / \mathrm{d}$ and for more than $5 \mathrm{~d}$. This exclusion rate was not greater than that in the International Children's Accelerometry Database, which found that only $62.2 \%$ of boys and $59.3 \%$ of girls in their study wore an accelerometer more than $12 \mathrm{~h} / \mathrm{d}$ over periods longer than $4 \mathrm{~d}^{(42)}$. On weekdays, members of the research team met with the subjects every morning to remind them to wear their accelerometers. However, TEE $E_{\text {DLW }}$ and $\mathrm{PAL}_{\mathrm{DLW}}$ were not different between the subjects included in the analysis and those who were excluded.

Fourth, we used a triaxial accelerometer made by a Japanese company. This made it difficult to compare the present results with those from Western countries, which have most often used Actigraph accelerometers. Actigraph accelerometers are not used widely in Japan, and Japanese people tend to be more familiar with pedometers and accelerometers made by Japanese companies. The present study examined the accuracy of the EW4800P accelerometer, and showed that it can estimate the total PA with a high degree of accuracy.

In conclusion, based on a comparison with the DLW method, the present study showed that EW4800P triaxial accelerometry can estimate daily TEE with good accuracy and precision. However, the accuracy of PAEE and PAL estimations was not high in Japanese adolescents. Prediction of RMR in Japanese adolescents and the prediction model of accelerometry for adolescents therefore need to be improved.

\section{Acknowledgements}

The authors thank the teachers, families and students who participated in and supported the study. The present study was supported by a Grant-in-Aid for Scientific Research (A) (no. 18200047) from the Ministry of Education, Culture, Sports, Science, and Technology, Japan. K. I.-T. analysed and interpreted the data, wrote the manuscript and had primary responsibility for the final content. K. Kaneko designed the main study protocol and managed the study. K. Koizumi managed the field study. C. I. managed the field study and $\mathrm{RMR}_{\mathrm{m}}$. All authors approved the final version of the manuscript. The authors declare that they have no conflicts of interest.

\section{References}

1. Ministry of Education Culture, Science, Sports (2010) School Health Statistics 2010. Tokyo: Ministry of Education, Culture, Science, Sports, Japan.

2. Watanabe M, Sasai Y, Miyake S, et al. (2008) A study of sports activity in the junior high school students. Okayama J Phys Educ 15, 13-18.

3. Adachi M, Sasayama K, Okisima K, et al. (2009) Assessing daily physical activity in junior high school students using pedometer with accelerometer. Jpn J Phys Fitness Sports Med 58, 275-284.

4. Hikihara Y, Saitoh S \& Yoshitake Y (2005) Validity of methods to measure total energy expenditure of baseball players in Japanese high school. Jpn J Phys Fitness Sports Med 54, 363-372.

5. Adachi M, Sasayama K, Hikihara Y, et al. (2007) Assessing daily physical activity in elementary school students used by accelerometer: a validation study against doubly labeled water method. Jpn J Phys Fitness Sports Med 56, 347-356.

6. Sirard JR \& Pate RR (2001) Physical activity assessment in children and adolescents. Sports Med 31, 439-454.

7. Tanaka C, Tanaka S, Kawahara J, et al. (2007) Triaxial accelerometry for assessment of physical activity in young children. Obesity (Silver Spring) 15, 1233-1241.

8. Chen X, Sekine M, Hamanishi S, et al. (2003) Validation of a self-reported physical activity questionnaire for schoolchildren. J Epidemiol 13, 278-287.

9. Yamada Y, Yokoyama K, Noriyasu R, et al. (2009) Lightintensity activities are important for estimating physical activity energy expenditure using uniaxial and triaxial accelerometers. Eur J Appl Physiol 105, 141-152.

10. International Atomic Energy Agency (2009) Assessment of Body Composition and Total Energy Expenditure in Humans using Stable Isotome Techniques, AEA Human Health Series No. 3, 19 October 2009, 133 pp. Vienna: International Atomic Energy Agency. 
11. Schoeller DA, Ravussin E, Schutz Y, et al. (1986) Energy expenditure by doubly labeled water: validation in humans and proposed calculation. Am J Physiol 250, R823-R830.

12. Racette SB, Schoeller DA, Luke AH, et al. (1994) Relative dilution spaces of ${ }^{2} \mathrm{H}$ - and ${ }^{18} \mathrm{O}$-labeled water in humans. Am J Physiol 267, E585-E590.

13. Weir JB (1949) New methods for calculating metabolic rate with special reference to protein metabolism. J Physiol 109, 1-9.

14. Black AE, Prentice AM \& Coward WA (1986) Use of food quotients to predict respiratory quotients for the doublylabelled water method of measuring energy expenditure. Hum Nutr Clin Nutr 40, 381-391.

15. Miyake Y, Sasaki S, Arakawa M, et al. (2008) Fatty acid intake and asthma symptoms in Japanese children: the Ryukyus Child Health Study. Clin Exp Allergy 38, 1644-1650.

16. Kobayashi S, Honda S, Murakami K, et al. (2012) Both comprehensive and brief self-administered diet history questionnaires satisfactorily rank nutrient intakes in Japanese adults. J Epidemiol 22, 151-159.

17. Ishikawa-Takata K, Tabata I, Sasaki S, et al. (2008) Physical activity level in healthy free-living Japanese estimated by doubly labelled water method and International Physical Activity Questionnaire. Eur J Clin Nutr 62, 885-891.

18. Lohman TG (1992) Advances in Body Composition. Champaign, IL: Human Kinetics.

19. Matsumura Y, Yamamoto M, Kitado T, et al. (2008) Highaccuracy physical activity monitor utilizing three-axis accelerometer. Natl Tech Rep 56, 60-66.

20. Ministry of Health Labour and Welfare, Japan (2010) Dietary Reference Intakes for Japanese, 2010. Tokyo: Ministry of Health, Labour, and Welfare, Japan.

21. Kumae T, Ito C, Koizumi K, et al. (2009) Study on accuracy of a hood system for measurement of resting energy expenditure using a phantom of respiratory system. J Phys Fit Nutr Immunol 19, 32-41.

22. Bland JM \& Altman DG (1995) Comparing methods of measurement: why plotting difference against standard method is misleading. Lancet 346, 1085-1087.

23. Bandini LG, Schoeller DA \& Dietz WH (1990) Energy expenditure in obese and nonobese adolescents. Pediatr Res 27 , 198-203.

24. Livingstone MBE, Prentice AM, Coward WA, et al. (1990) Total energy expenditure during childhood and adolescence. Proc Nutr Soc 50, 1991.

25. Bratteby LE, Sandhagen B, Fan H, et al. (1998) Total energy expenditure and physical activity as assessed by the doubly labeled water method in Swedish adolescents in whom energy intake was underestimated by 7-d diet records. Am J Clin Nutr 67, 905-911.

26. Perks SM, Roemmich JN, Sandow-Pajewski M, et al. (2000) Alterations in growth and body composition during puberty. IV. Energy intake estimated by the youth-adolescent foodfrequency questionnaire: validation by the doubly labeled water method. Am J Clin Nutr 72, 1455-1460.
27. Ekelund U, Aman J, Yngve A, et al. (2002) Physical activity but not energy expenditure is reduced in obese adolescents: a case-control study. Am J Clin Nutr 76, 935-941.

28. Slinde F, Arvidsson D, Sjoberg A, et al. (2003) Minnesota leisure time activity questionnaire and doubly labeled water in adolescents. Med Sci Sports Exerc 35, 1923-1928.

29. DeLany JP, Bray GA, Harsha DW, et al. (2004) Energy expenditure in African American and white boys and girls in a 2-y follow-up of the Baton Rouge Children's Study. Am J Clin Nutr 79, 268-273.

30. Spadano JL, Bandini LG, Must A, et al. (2005) Longitudinal changes in energy expenditure in girls from late childhood through midadolescence. Am J Clin Nutr 81, 1102-1109.

31. Ekelund U, Sarnblad S, Brage S, et al. (2007) Does physical activity equally predict gain in fat mass among obese and nonobese young adults? Int J Obes (Lond) 31, 65-71.

32. Eiholzer U, Meinhardt U, Rousson V, et al. (2008) Association between short sleeping hours and physical activity in boys playing ice hockey. J Pediatr 153, 640-645, 645.e1.

33. Corder K, van Sluijs EM, Wright A, et al. (2009) Is it possible to assess free-living physical activity and energy expenditure in young people by self-report? Am J Clin Nutr 89, 862-870.

34. Ito C, Koizumi K, Atsumi K, et al. (2007) The relationship between ultrasound calaneal bone mass and lifestyle, physical fitness and body composition in junior high school students. J Jpn Soc Nutr Food Sci 60, 53-59.

35. Park J, Ishikawa-Takata K, Tanaka S, et al. (2011) Effects of walking speed and step frequency on estimation of physical activity using accelerometers. J Physiol Anthropol 30, 119-127.

36. Tudor-Locke C, Craig CL, Beets MW, et al. (2011) How many steps/day are enough for children and adolescents? Int $J$ Behav Nutr Phys Act 8, 78.

37. Corder K, Brage S, Wright A, et al. (2010) Physical activity energy expenditure of adolescents in India. Obesity (Silver Spring) 18, 2212-2219.

38. Cooper JA, Watras AC, O'Brien MJ, et al. (2009) Assessing validity and reliability of resting metabolic rate in six gas analysis systems. J Am Diet Assoc 109, 128-132.

39. de Graauw SM, de Groot JF, van Brussel M, et al. (2010) Review of prediction models to estimate activity-related energy expenditure in children and adolescents. Int J Pediatr 2010, 489304

40. Corder K, Brage S, Wareham NJ, et al. (2005) Comparison of PAEE from combined and separate heart rate and movement models in children. Med Sci Sports Exerc 37, 1761-1767.

41. Strath SJ, Pfeiffer KA \& Whitt-Glover MC (2012) Accelerometer use with children, older adults, and adults with functional limitations. Med Sci Sports Exerc 44, 1 Suppl. 1, S77-S85.

42. Sherar LB, Griew P, Esliger DW, et al. (2011) International children's accelerometry database (ICAD): design and methods. BMC Public Health 11, 485. 